\title{
Prediction of lethal and synthetically lethal knock-outs in regulatory networks
}

\author{
Gunnar Boldhaus, Florian Greil, Konstantin Klemm
}

the date of receipt and acceptance should be inserted later

\begin{abstract}
The complex interactions involved in regulation of a cell's function are captured by its interaction graph. More often than not, detailed knowledge about enhancing or suppressive regulatory influences and cooperative effects is lacking and merely the presence or absence of directed interactions is known. Here we investigate to which extent such reduced information allows to forecast the effect of a knock-out or a combination of knock-outs. Specifically we ask in how far the lethality of eliminating nodes may be predicted by their network centrality, such as degree and betweenness, without knowing the function of the system. The function is taken as the ability to reproduce a fixed point under a discrete Boolean dynamics. We investigate two types of stochastically generated networks: fully random networks and structures grown with a mechanism of node duplication and subsequent divergence of interactions. On all networks we find that the out-degree is a good predictor of the lethality of a single node knock-out. For knock-outs of node pairs, the fraction of successors shared between the two knockedout nodes (out-overlap) is a good predictor of synthetic lethality. Out-degree and out-overlap are locally defined and computationally simple centrality measures that provide a predictive power close to the optimal predictor.
\end{abstract}

\section{G. Boldhaus}

Bioinformatics Group, Institute for Computer Science, Universität Leipzig, Härtelstraße 16-18, 04107 Leipzig, Germany

F. Greil

Alfred Wegener Institute for Polar and Marine Research, Am Handelshafen 12, 27570 Bremerhaven, Germany

\section{K. Klemm}

Bioinformatics Group, Institute for Computer Science, Universität Leipzig, Härtelstraße 16-18, 04107 Leipzig, Germany E-mail: klemm@bioinf.uni-leipzig.de
Keywords knock-out; synthetic lethality; prediction; network centrality; Boolean network

\section{Introduction}

The survival, functioning and growth of a living cell is based on a large set of interdependent biochemical interactions. Interaction networks (Bower and Bolouri 2001) have proven to be useful summary pictures of such a biochemical system or part of it, especially when interactions are known qualitatively while precise quantitative information is scarce. For many systems, the interaction network suffices to capture essential features of dynamical behaviour (Albert and Othmer, 2003) such as the presence of a stable stationary state, multistability, oscillations etc. Often such predictions do not even depend on the whole network structure. Qualitative statements on system behaviour may be based on the centrality of nodes (Jeong et al., 2001, Wuchty and Stadler, 2003) or the presence of certain small subnetworks, called motifs (Alon, 2007).

Here we ask to what extent a limited knowledge of biochemical interactions is usable for predicting the reaction of a system to failure of one or several of its components (Albert et al. 2000, Inger et al. 2009, Boldhaus et al., 2010). This kind of theory serves to complement experiments with knock-outs in vivo or in vitro (de Visser et al. 2003). A knock-out (or knock-down) is performed by blocking (or reducing) production of a single protein. Depending on the viability of the cell after suffering the modification, knock-outs are subject to a binary classification into lethal and viable.

When knocking out several nodes (proteins) of a system simultaneously, a richer classification arises from considering the lethality of this combined knock-out 
together with the effect of each single knock-out. Synthetic lethality (Hartman et al., 2001) is the class of lethal simultaneous knock-out of two nodes $i$ and $j$, where independent knock-out of node $i$ alone is viable and independent knock-out of node $j$ alone is viable. Synthetic lethality has direct implications for target identification in anticancer drug discovery (Chan and Giaccia, 2011). Since the experimental effort of a complete scan of double knock-outs is quadratic in the number of proteins, an accurate computational prediction of candidate pairs can greatly reduce the cost of experiments.

Here we study prediction of lethality and synthetic lethality in stochastically generated interaction networks. Knowledge is taken to be incomplete in the sense that only the absence or presence of interactions but not the type (enhancer / suppressor) is available. As predictors, we test efficiently computable network centrality measures based on degree and betweenness. Quality of predictors in terms of ROC curves (see Section 6) is held against the optimal prediction possible with the available knowledge. Additionally, we use evolutionary distance between nodes as a predictor. Thereby we find out how much the knowledge of paralogs supports the identification of synthetically lethal pairs.

Our notion of viability and lethality is based on a functional phenotype that we define here as a stationary state of the unperturbed dynamical system. Regulatory interactions are mimicked by Boolean threshold dynamics that serves as a suitable testbed for the studies of robustness of networked biological systems (Bornholdt, 2005 ) and for evolutionary studies (Luo and Turner, 2011).

\section{Network construction}

Throughout this contribution, a network on $n$ nodes is given by an $n \times n$ matrix $W$. Each matrix entry $w_{i j}$ takes a value in $\{-1,0,+1\}$ where

$w_{i j}=\left\{\begin{array}{c}+1, \text { if } j \text { is an enhancer of } i \\ -1, \text { if } j \text { is a suppressor of } i \\ 0, \text { otherwise }\end{array}\right.$.

Reduced information about interactions is represented by assigning the network $W$ a directed graph that we identify with its adjacency matrix $A$. The entries of $A$ are given by $a_{j i}=\left|w_{i j}\right|$. Thus $A$ contains the information about the absence or presence but not the type of a directed interaction. The density $\rho(W)$ of a network

$\rho(W)=n^{-2} \sum_{i=1}^{n} \sum_{j=1}^{n}\left|w_{i j}\right|$

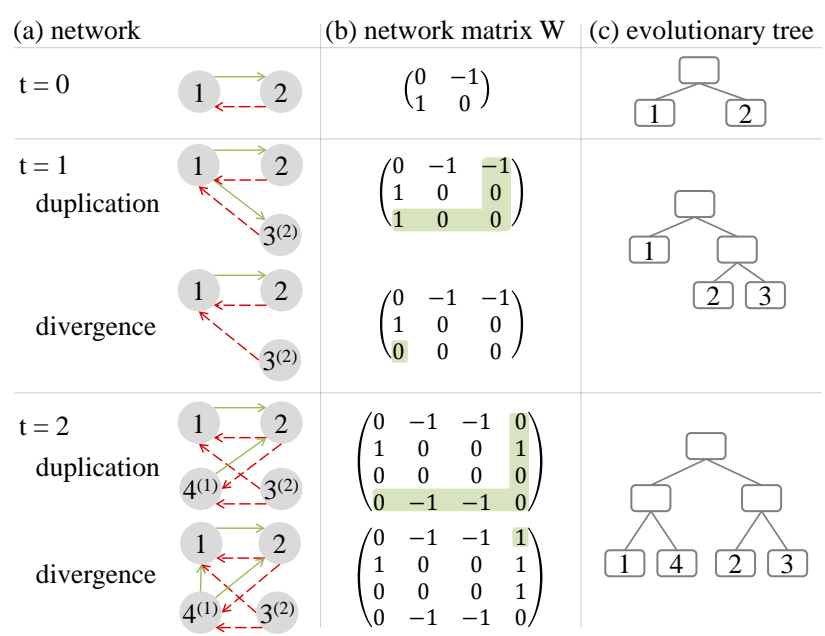

Fig. 1 Example of network generation of a network with duplication and divergence. (a) The network at time-step $t=0$ is initialized with two asymmetrically coupled nodes. Afterwards it grows by successive duplication and divergence steps. (b) The growth process in terms of the network matrix $W$. (c) In the evolutionary tree, each leaf represents an extant node in the network. Inner nodes are common ancestors.

measures the fraction of interactions established out of the $n^{2}$ possible ones. We generate networks with the following two stochastic procedures.

\subsection{Random networks}

A random network $W$ is generated by independently assigning each entry $w_{i j}$ a value +1 with probability $p / 2$, a value -1 with probability $p / 2$ and a value 0 with probability $1-p$. The model parameter $p$ is to be chosen from $[0,1]$ and determines the average density of the random network (Drossel, 2008, Aldana et al. 2003 ).

\subsection{Networks from duplication and divergence}

An alternative statistical ensemble of networks is generated by duplication and divergence. This is motivated by the observation that an evolutionary extension of the repertoire of regulatory sequences is obtained by duplication (Wagner, 1994; Solé et al. 2002 , Pastor-Satorras et al. 2003; Ispolatov et al., 2005; Aldana et al., 2007).

For generating a network by duplication and divergence $(\mathrm{DaD})$ we start with a $2 \times 2$ matrix representing two mutually but unequally coupled nodes, i.e. $w_{12}=+1, w_{21}=-1$ and $w_{11}=w_{22}=0$. Then the following process of duplication (i) and divergence (ii) is iterated.

(i) A node $i$ of the network with $n-1$ nodes is randomly drawn from a flat probability distribution. Node $i$ 

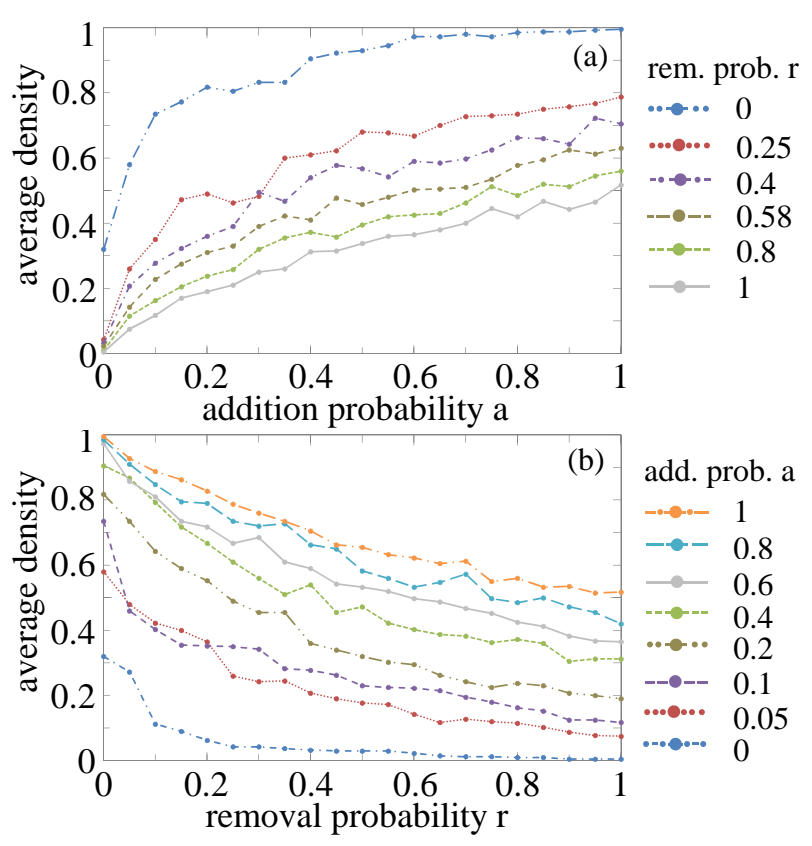

Fig. 2 Average densities for networks generated with duplication and divergence. (a) Average density with fixed removal probabilities $r$. (b) Average density with fixed addition probabilities $a$. All points are averages over $10^{6}$ realizations of networks with $n=20$ nodes.

is duplicated, generating an additional row and column with index $n$ in the matrix $W$. The new entries are $w_{j, n}:=w_{j, i}$ and $w_{n, j}:=w_{i, j}$ for all $1 \leq j<n$, and $w_{n, n}=w_{i, i}$.

(ii) For each index pair $(k, l)$ with $k=n$ or $l=n$ : if $\left|w_{k l}\right|=1$, we set $w_{k l}:=0$ with probability $r$ and leave $w_{k l}$ unchanged with probability $1-r$. Otherwise $\left(w_{k l}=0\right)$, we set $w_{k l}:=+1$ with probability $a / 2, w_{k l}:=-1$ with probability $a / 2$ and leave $w_{k l}$ unchanged with probability $1-a$.

Step (i) implements gene duplication, in which both the original and the replicated proteins retain the same structural properties and the same set of interactions. The divergence steps (ii) implements the possible mutations of the replicated gene, which translate into the addition and removal of interactions with probabilities $a$ and $r$. An example of this process is shown in Figure 1. Special attention is given to the handling of loops. If the randomly chosen original node has a loop, the loop is copied as well as two additional links with the same edge weight between the original and the replica node.

The average density of networks generated with duplication and divergence is shown in Figure 2 as a function of parameters $a$ and $r$.

\section{Knock-outs, dynamics, and functionality}

\subsection{Knock-outs}

In a real biochemical interaction network, knocking out a node means that the concentration of the reactant represented by the node is set zero. For our purposes, it is equivalent to remove all outgoing interactions (arcs) of the node from the network. The knock-out of node $k$ in network $W$ leaves the network as $W^{\backslash\{k\}}$ with matrix entries $w_{i j}^{\backslash\{k\}}=w_{i j}$ if $j \neq k$ and 0 otherwise. As a generalization, several nodes forming a set $K \subset\{1,2, \ldots, n\}$ may be knocked out. The resulting network $W^{\backslash K}$ has entries

$w_{i j}^{\backslash K}= \begin{cases}w_{i j} & \text { if } j \notin K \\ 0 & \text { otherwise. }\end{cases}$

\subsection{Dynamics}

In the present work we model gene regulatory networks by threshold dynamics (Derrida, 1987). This is a special case of Boolean dynamics (Kauffman, 1969, Aldana et al. 2003). A multitude of formulations for Boolean threshold networks exist, depending how the thresholds are distributed, how the behaviour at the threshold is defined and which weights for the edges are allowed. We choose the version as applied in the simplified yeast cell cycle network (Li et al., 2004 ) and many succeeding studies (Boldhaus and Klemm, 2010; Boldhaus et al. 2010, Szejka et al., 2008, Davidich and Bornholdt 2008), compare Equation 4 .

A node is activated, $s_{i}=1$, if the sum of its weighted inputs exceeds a threshold assumed to be zero here. It is deactivated if the input sum falls below the threshold. In the case when the sum gives exactly the threshold value, the node value remains unchanged. Thus the Boolean state $s_{i}$ of node $i$ at time step $t$ evaluates to

$s_{i}(t+1)=\left\{\begin{array}{rl}1 & \text { if } \sum_{j=1}^{n} w_{i j} s_{j}(t)>0 \\ 0 & \text { if } \sum_{j=1}^{n} w_{i j} s_{j}(t)<0 \\ s_{i}(t) & \text { if } \sum_{j=1}^{n} w_{i j} s_{j}(t)=0\end{array}\right.$.

This threshold dynamics does not capture the wealth of combinatorial effects implementable by control at the transcriptional level (Buchler et al., 2003). However, it is able to account both for cooperative and noncooperative interactions. In a network with $w_{i j}=w_{i k}=$ 1 being the only incoming connections of node $i$, for instance, these two inputs $j$ and $k$ act non-cooperatively on node $i$. Then $s_{j}(t)=1 \vee s_{k}(t)=1$ is sufficient for $s_{i}(t+1)=1$, amounting to an oR operation. 


\subsection{Functionality and lethality}

A state $s^{*}$ is a fixed point if it remains unaltered by the dynamics, i.e. the successor state of $s^{*}$ is $s^{*}$ itself. We define the function (in the sense of a phenotype) of a network $W$ to be a fixed point state $s^{*} \neq(0, \ldots, 0)$. After generation of a network, a fixed point $s^{*}$ is found as described in Section 6

Given a network $W$ and its functional fixed point $s^{*}$, we say that a knock-out $K \subset\{1, \ldots, n\}$ is viable (for $W, s^{*}$ ) if $s^{*}$ is a fixed point of $W^{\backslash K}$. Otherwise $K$ is lethal. Note that a sufficient (but not necessary) condition for $K$ to be viable is that $s_{i}^{*}=0$ for all $i \in K$ : a lethal effect is not caused by knocking out nodes that are inactive already.

We say that $K$ is synthetically lethal, if

(i) $K$ is lethal and

(ii) $K^{\prime}$ is viable for all proper subsets $K^{\prime} \subset K$.

Analogously one may define synthetic viability. A knockout $K$ is synthetically viable, if

(i) $K$ is viable and

(ii) $K^{\prime}$ is lethal for all proper subsets $K^{\prime} \subset K, K^{\prime} \neq \emptyset$.

Thus synthetic lethality and synthetic viability are defined for arbitrary cardinality $|K| \geq 2$ of knock-outs. In this contribution, however, only single and double knock-outs are considered.

One might wonder in which sense our definitions match a possibly more intuitive definition of a knockout. Naturally, a knock-out of a node $i$ could be defined as a modification of the dynamical rules, Eq. (4), where we keep $s_{i}$ at value zero, irrespective of the input signals node $i$ receives. Then for such a knock-out to be called viable, we would require that this modified dynamics has a fixed point $r^{*}$ with $r_{j}^{*}=s_{j}^{*}$ for $j \neq i$ and $r_{i}^{*}=0$ otherwise. Let us compare this to the above definitions. Rather than changing the dynamical rules, the network itself is modified by removing all outgoing interactions of the node knocked out. On all other nodes, this has the same effect as keeping $s_{i}$ at state zero. Then, if the dynamics has a fixed point that coincides with $s^{*}$ on all nodes $j \neq i$, also node $i$ will be in state $s_{i}^{*}$ at this fixed point. Hence the present definitions, while being convenient and concise in notation, coincide with the intuitive notion.

\section{Results}

All results presented in this section are based on simulations with networks having $n=20$ nodes. The Supplementary Material provides results for larger and smaller networks for comparison.

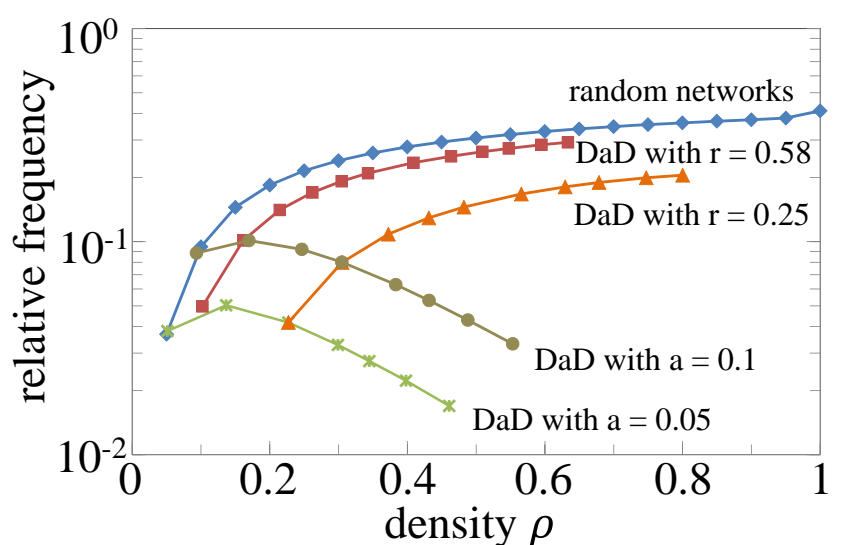

Fig. 3 Probability of lethal single node knock-outs as a function of network density $\rho$. All values are averages over $10^{6}$ realizations at the given value of $\rho$. Holding the addition (removal) probability $a(r)$ constant limits the interval of possible densities.

\subsection{Statistics of lethal knock-outs}

We start by presenting the effect of single knock-outs in the two models of networks (random and DaD). Figure 3 shows, as a function of the density of the network, the probability that a single knock-out is lethal. For random networks, this probability increases with the arc density $\rho$. For all choices of parameters investigated here, DaD networks have fewer lethal knock-outs than random networks at the same density. Under constant probability $a$ of adding interactions, the DaD networks even exhibit a decreasing number of lethal knock-outs with increasing density.

Note that the probability of knock-outs being lethal cannot exceed $1 / 2$ because on average half of the nodes are in the off-state on the functional fixed point. Knockout of a node in the off-state in the network does not affect the states of the other nodes. This theoretical maximum, however, is not reached. Random networks at the largest possible density 1 have a probability of $\approx 0.41$ for a knock-out to be lethal.

Now we turn to the statistics for double knock-outs $\{v, w\}$ in combination with the single knock-outs $\{v\}$ and $\{w\}$. In each of the panels of Figure 4, the open circles connected by solid curves give the fraction of synthetically lethal pairs of nodes in networks of a given density $\rho$. Synthetic lethality becomes more abundant with increasing density in random networks (top panel) and in DaD networks with fixed arc removal probability (two lower panels).

Synthetic lethality is just one possible outcome of knock-out tests performed on a pair $\{v, w\}$. Of the singlenode knock-outs $\{v\}$ and $\{w\}$, none, exactly one or both may be lethal. Combination of this ternary result with the binary outcome (lethal/ viable) of the 
Table 1 Overview of the area under the ROC curves for prediction of single node knock-outs. Each pair of rows is for networks with a given expected density $p$. The first row of each pair refers to random networks with parameter value $p$. The second row of each pair is for DaD networks with parameter values $a$ and $r$. Higher values of the area refer to a higher accuracy of the prediction.

\begin{tabular}{lcccccc}
\hline & struct. lethality & out-deg. & out + in-deg. & out - in-deg. & betw.centr. & in-deg. \\
\hline \hline$p=0.14$ & 0.672 & 0.649 & 0.621 & 0.591 & 0.566 & 0.483 \\
$a=0.05, r=0.58$ & 0.732 & 0.708 & 0.634 & 0.662 & 0.593 & 0.486 \\
$p=0.23$ & 0.623 & 0.612 & 0.587 & 0.572 & 0.560 & 0.492 \\
$a=0.1, r=0.58$ & 0.681 & 0.666 & 0.608 & 0.626 & 0.577 & 0.494 \\
$p=0.26$ & 0.612 & 0.602 & 0.579 & 0.566 & 0.557 & 0.494 \\
$a=0.05, r=0.25$ & 0.689 & 0.682 & 0.635 & 0.617 & 0.598 & 0.534 \\
$p=0.35$ & 0.588 & 0.581 & 0.562 & 0.553 & 0.548 & 0.496 \\
$a=0.1, r=0.25$ & 0.648 & 0.642 & 0.602 & 0.596 & 0.574 & 0.518 \\
\hline
\end{tabular}

Table 2 Overview of the area under the ROC curves for prediction of double node knock-outs which exhibit synthetic lethality. Each pair of rows is for networks with a given expected density $p$. The first row of each pair refers to random networks with parameter value $p$. The second row of each pair is for DaD networks with parameter values $a$ and $r$. Prediction based on evolutionary distance is only applicable for networks generated with duplication and divergence. Results which incorporate prior knowledge of the result of single node knock-outs are shown in brackets. Higher values of the area refer to a higher accuracy of the prediction.

\begin{tabular}{|c|c|c|c|c|c|}
\hline & struct. syn. let. & out-overlap & repl. centr. & evol. distance & in-overlap \\
\hline$p=0.14$ & $0.888(0.895)$ & $0.859(0.865)$ & $0.597(0.600)$ & - & $0.500(0.499)$ \\
\hline$a=0.05, r=0.58$ & $0.915(0.922)$ & $0.896(0.903)$ & $0.594(0.597)$ & $0.601(0.601)$ & $0.531(0.530)$ \\
\hline$p=0.23$ & $0.778(0.787)$ & $0.742(0.752)$ & $0.582(0.586)$ & - & $0.501(0.500)$ \\
\hline$a=0.1, r=0.58$ & $0.857(0.867)$ & $0.832(0.841)$ & $0.588(0.591)$ & $0.550(0.551)$ & $0.519(0.519)$ \\
\hline$p=0.26$ & $0.743(0.752)$ & $0.705(0.717)$ & $0.576(0.581)$ & - & $0.500(0.500)$ \\
\hline$a=0.05, r=0.25$ & $0.799(0.812)$ & $0.779(0.789)$ & $0.605(0.609)$ & $0.613(0.611)$ & $0.573(0.572)$ \\
\hline$p=0.35$ & $0.673(0.681)$ & $0.632(0.646)$ & $0.559(0.563)$ & - & $0.500(0.500)$ \\
\hline$a=0.1, r=0.25$ & $0.735(0.748)$ & $0.707(0.724)$ & $0.583(0.587)$ & $0.570(0.568)$ & $0.546(0.546)$ \\
\hline
\end{tabular}

two-node knock-out $\{v, w\}$ yields six possible scenarios. The statistics of these scenarios is plotted in Figure 4. Interestingly, synthetic viability (dotted line with triangles) becomes more abundant than synthetic lethality in dense random networks.

\subsection{Prediction}

Now we investigate the predictability of the lethality, first of single, then of double knock-outs. Predictability is strongly dependent on the available knowledge. If the network $W$ and the functional fixed point $s^{*}$ are fully known, perfect prediction of lethality is possible simply by computing the effect of the knock-out. In a more realistic scenario, only partial knowledge is available which we model here as follows. The presence or absence of each interaction is available with absolute accuracy while the type (enhancer/ suppressor) of each present interaction is totally unknown; information on the functional fixed point is not available. With the formalism described in section 2 for a network $W$ only the adjacency matrix $A$ of the directed graph is known. Then the best predictor of lethality of a knock-out $\{i\}$ is the relative frequency $l_{i}$ of $\{i\}$ being lethal in networks $W^{\prime}$ that have adjacency matrix $A$ and interaction types randomly assigned. In other words, $\{i\}$ is predicted as lethal in $W$, if $\{i\}$ is typically lethal in networks with the same adjacency matrix as $W$. We call $l_{i}$ structural lethality.

Computation of $l_{i}$ may be impossible or impractical in real scenarios. Therefore the value of a centrality measure at node $i$ is extracted from $A$ and used for prediction instead of $l_{i}$. This incurs another step of knowledge reduction. Here it is salient to choose the "right" centrality measure for prediction. We consider the betweenness centrality $b_{i}$, the out-degree $d_{i}^{\text {out }}$ the in-degree $d_{i}^{\text {in }}$, furthermore the degree sum $d_{i}^{\text {out }}+d_{i}^{\text {in }}$ and the degree difference $d_{i}^{\text {out }}-d_{i}^{\text {in }}$. These are defined in Section 6 as well as other quantities used here. The predictive power of the different measures is summarized in Table 1 for random and DaD models with varied parameter values. The deviation of a value from 0.5 indicates that the quantity contains information about the lethality of nodes in the given scenario. This is the case for all the centrality measures under consideration except for the in-degree. In DaD networks the chance 

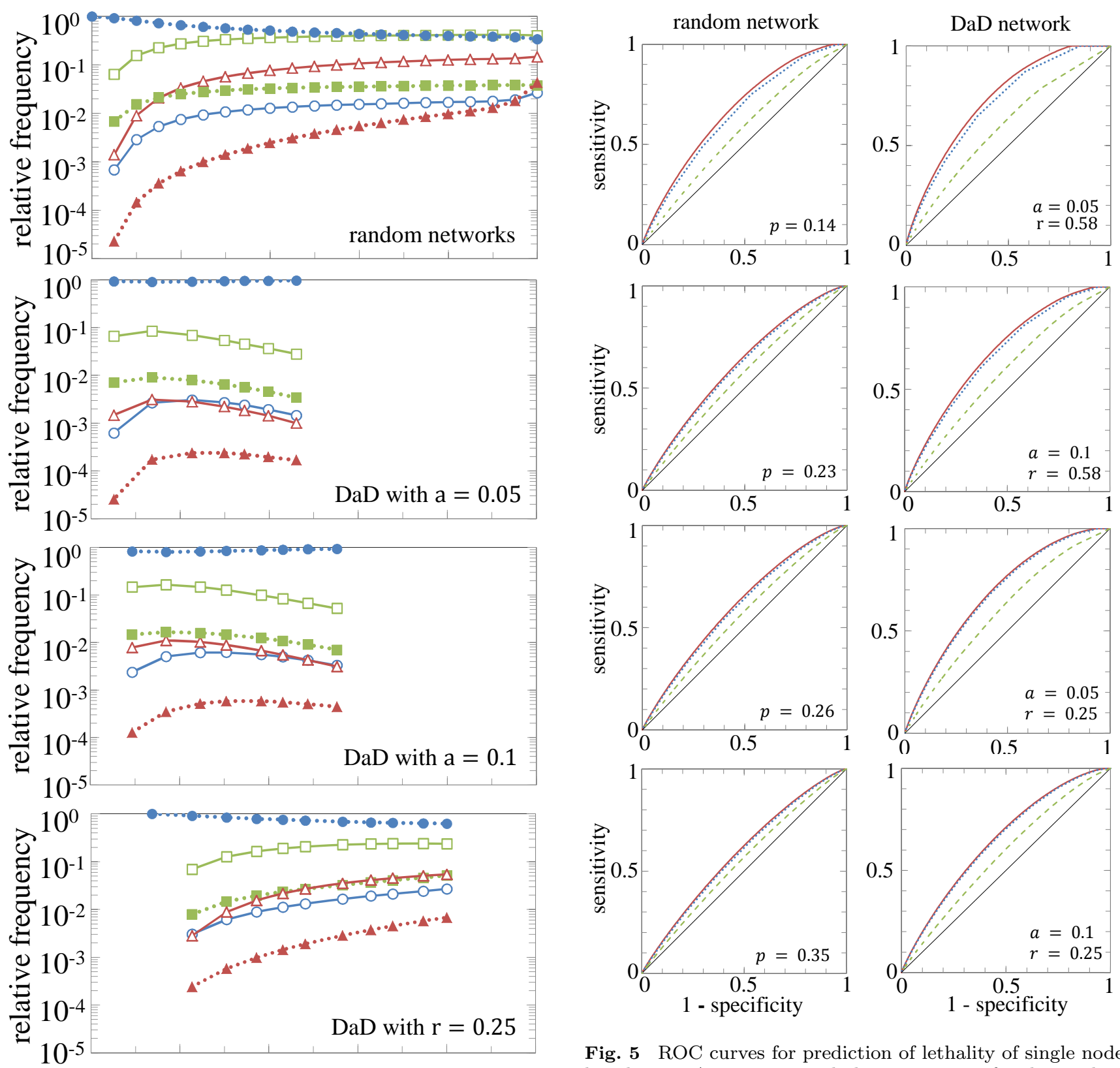

Fig. 5 ROC curves for prediction of lethality of single node knock-outs. Average network density is $\rho=p$ for the random networks (panels in left column). In the corresponding panel in the right column, results for DaD networks with the same density are shown. Predictors are structural lethality (solid curve, optimal predictor), out-degree (dotted), and betweenness centrality (dashed). The solid diagonal is the line of no discrimination. Each curve is based on $10^{4}$ network realizations. The synthetic lethality is estimated by extracting the graph from each network and probing another $10^{3}$ network realizations with the same graph structure.

DaD with $r=0.58$

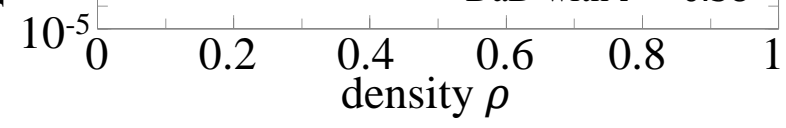

Fig. 4 Lethality of knock-outs as a function of network density $\rho$. Open symbols refer to lethal double knock-outs while solid symbols mean that the double knock-out is still viable. The shape of the symbols distinguishes between results of single-node knock-outs: neither single knock-out (circle), only one knock-out (square) or both single knock-outs are lethal (triangle). All values are averages over $10^{6}$ realizations at the given value of $\rho$. 

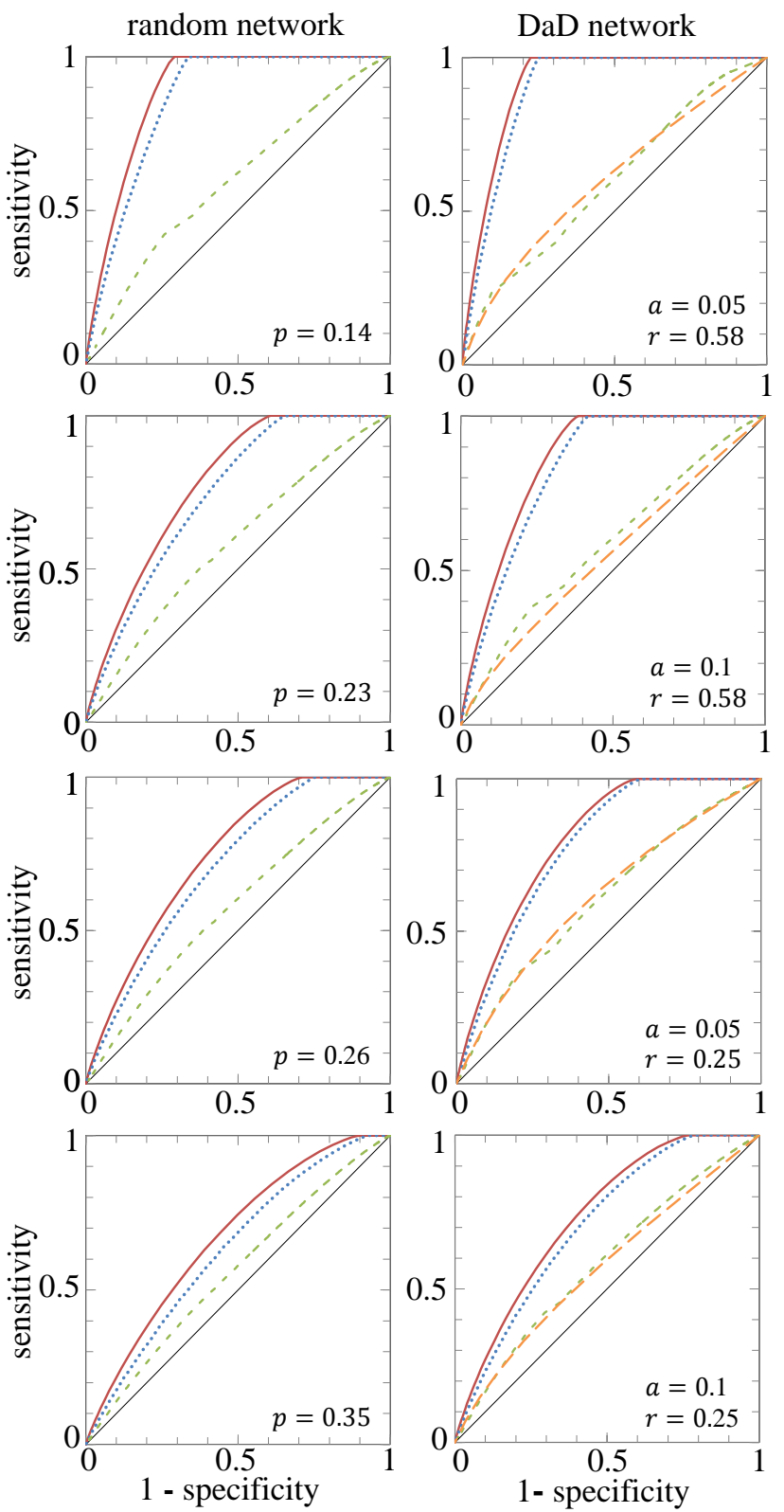

Fig. 6 ROC curves for prediction of synthetic lethality. Average network density is $\rho=p$ for the random networks (panels in left column). In the corresponding panel in the right column, results for DaD networks with the same density are shown. Predictors are structural synthetic lethality (solid curve, optimal predictor), out-overlap (dotted), replacement centrality (short-dashed), and evolutionary distance (longdashed). The solid diagonal is the line of no discrimination. Each curve is based on $10^{4}$ network realizations. The structural synthetic lethality is estimated by extracting the graph from each network and probing another $10^{3}$ network realizations with the same graph structure. to predict the effect of a knock-out is larger than in random networks of the same density, e.g. $a=0.1, r=0.25$ leads to networks with density $p \approx 0.35$ which show a larger predictability than random networks with the same density. Best predictions are based on the outdegree whose predictive power almost reaches the best possible obtained by the structural lethality. Predictive power is measured as the area under the ROC curve of sensitivity versus specificity of the prediction which are shown in Figure 5.

Now we study the prediction of synthetically lethal pairs. The framework is mostly analogous to that of single knock-outs. Again we assume that the adjacency matrix but not the full network (with interaction types) are known. Eligible predictors are now measures of joint centrality, i.e. mapping a given unordered node pair $\{i, j\}$ in a given graph $A$ to a number. Here we investigate the out-overlap $o_{i j}^{\text {out }}$, the in-overlap $o_{i j}^{\text {in }}$ and the replacement centrality $r_{i j}$ as defined in section 6. Furthermore, the evolutionary distance $e_{i j}$ is used as a predictor in networks evolved by duplication and divergence (DaD). Table 2 summarizes the predictive power of these centrality measures, again in comparison with that of the optimal predictor $s_{i j}$ here called structural synthetic lethality. As the main result, the out-overlap $o_{i j}^{\text {out }}$ is the best predictor of synthetic lethality in all cases considered, its predictive power is close to optimal in all cases considered. Prediction of synthetic lethality is facilitated in DaD networks as compared to random networks.

An interesting alternative scenario arises under the assumption that we already know all viable single node knock-outs, $V(W)=\{i:\{i\}$ viable $\}$ in each network $W$ considered. For the prediction of synthetically lethal pairs, candidates are subsets $\{i, j\} \subseteq V(W), i \neq j$. The predictive power for this scenario with restricted candidate set is given in brackets in Table 2. Prior knowledge of viable single-node knock-outs does not induce a significant increase of predictive power for any of the combinations of predictor, network generation model and density.

For a more detailed view of sensitivity and specificity, Figure 6 shows the ROC curves of selected predictors in the case without prior knowledge of viable single knock-outs.

\section{Concluding remarks}

The present contribution has established a theoretical framework for assessing predictability of knock-out effects in networked regulatory systems. We covered a broad range of scenarios in terms of network structures and predictors. Results are robust under variation of 
system size and choice of functional fixed points, cf. additional results in Supplementary Material.

Nevertheless it must be emphasized that outcomes depend on the choice of specific definitions made. First of all, the definition of lethality is made in the context of the regulatory network, assuming that the only task of a node is regulation within the system considered. If the disabled protein is involved otherwise, e.g. as a structural protein, its potentially lethal knock-out cannot be predicted in the present framework. Secondly, the definition of functionality of the network as the presence of a fixed point is not the only reasonable choice. For instance, we may demand that the fixed point $s^{*}$ be stable in the sense that the system returns to $s^{*}$ after a perturbation at one node's state. Rather than a fixed point, a particular temporal sequence of states may be defined as the functionality of the system ( $\mathrm{Li}$ et al. 2004 Davidich and Bornholdt, 2008, Boldhaus et al. 2010 Luo and Turner, 2011).

The aim of the present study is to contribute to the theoretical background of knock-out experiments. It elucidates in how far the lethal effect of knock-outs is predictable by efficiently computable measures of node centrality. A future extension may be concerned with the effect of disabling or modifying single regulatory interactions rather than entirely knocking out genes. Such a scenario corresponds to natural or experimentally induced mutations of transcription factor binding sites. Analogous to the present study, a comparison of measures of edge centrality can find the best predictors for the lethality of such mutations. Alternatively, the present scenario using node centralities may be applied to the line graph of the regulatory network.

\section{Methods}

\subsection{Finding functional fixed point}

After generating a network $W$, the functional fixed point $s^{*}$ is assigned as follows. An initial state vector $s(0) \in$ $\{0,1\}^{n}$ is drawn uniformly. The dynamics is run from $s(0)$ by iterating Equation (4) until a state is seen the second time and an attractor is reached, i.e. at times $t_{1}>t_{2} \geq 0$ such that $s\left(t_{1}\right)=s\left(t_{2}\right)$. If the attractor is a non-trivial fixed point, $s\left(t_{2}\right)=s\left(t_{2}-1\right) \neq(0,0, \ldots, 0)$, we take it as the functional fixed point $s^{*}:=s\left(t_{2}\right)$. Otherwise the network $W$ is discarded and replaced by another random instance. This procedure preferentially chooses functional fixed points with larger attractor basins.

For comparison, the Supplementary Material provides additional results obtained by a different procedure for choosing the functional fixed point. We first determine the set $\left.F \subseteq\{0,1\}^{n} \backslash\{0, \ldots, 0)\right\}$ of fixed points of the given network $W$. If $F$ is not empty, the functional fixed point $s^{*}$ is drawn uniformly from $F$. Otherwise the network $W$ is discarded and replaced by another random instance.

\subsection{Evolutionary distance}

Along with the generation of a network $W$ with the $\mathrm{DaD}$ model (Section 2.2), the evolutionary tree $T$ is constructed, cf. the example in Figure 1. The nodes $\{1,2, \ldots, n\}$ of the network $W$ are the leaves of the tree $T$. The evolutionary distance $e_{i j}$ is defined as the length of the path between leaves $i$ and $j$ on $T$. Each edge on $T$ is counted with unit length.

\subsection{Measures of centrality}

Degree centralities They measure importance in a linear fashion, assuming that a node with twice the number of links also is twice as important. Several different degree centralities can be defined by using a function of the in-degree $d_{i}^{\text {in }}$ and the out-degree $d_{i}^{\text {out }}$ of a node $i$ for a given adjacency matrix $A$ :

$d_{i}^{\text {in }}(A)=\sum_{j=1}^{n} a_{j i} \quad d_{i}^{\text {out }}(A)=\sum_{j=1}^{n} a_{i j}$.

Overlaps We distinguish the in-overlap $o^{\text {in }}$ between nodes $i$ and $j$ as

$o_{i j}^{\text {in }}(A)=\frac{\left|\left\{k: a_{k i}=1 \wedge a_{k j}=1\right\}\right|}{\left|\left\{k: a_{k i}=1 \vee a_{k j}=1\right\}\right|}$

and the out-overlap $o^{\text {out }}$

$o_{i j}^{\text {out }}(A)=\frac{\left|\left\{k: a_{i k}=1 \wedge a_{j k}=1\right\}\right|}{\left|\left\{k: a_{i k}=1 \vee a_{j k}=1\right\}\right|}$

If the denominator is zero, the whole expression is defined to be zero.

Betweenness centrality It quantifies the fraction of shortest paths that pass through this node (Freeman, 1977).

$b_{i}(A)=\sum_{(j, k)} \frac{\sigma_{j k}(i)}{\sigma_{j k}}$,

where the sum runs over all ordered node pairs $(j, k)$; $\sigma_{j k}$ denotes the total number of shortest paths from node $j$ to node $k ; \sigma_{j k}(i)$ is the number of such paths running through node $i$. A modified Floyd-Warshall algorithm (Brandes, 2001) allows to simultaneously compute the lengths and numbers of shortest paths. 
Replacement centrality Let us define the replacement centrality of a pair of nodes $(i, j)$ as

$r_{i j}=\frac{b_{i}\left(A^{\backslash\{j\}}\right)+b_{j}(A \backslash\{i\})}{b_{i}(A)+b_{j}(A)}$

if the denominator in Equation (9) is non-zero and $r_{i j}(A)=0$ otherwise.

Structural synthetic lethality When only knowing the graph $A$, the best predictor of synthetic lethality for nodes $i$ and $j$ is given by the fraction of networks exhibiting synthetic lethality at $i$ and $j$ out of all networks compatible with $A$. This fraction is called structural synthetic lethality and is formally defined as

$s_{i j}(A)=\frac{\mid\{W \in \mathcal{N}(A) \mid\{i, j\} \text { synth. lethal in } W\} \mid}{|\mathcal{N}(A)|}$

where $\mathcal{N}(A)$ is the set of all networks that map to the graph $A$.

\subsection{Receiver Operating Characteristic (ROC)}

The sensitivity of a prediction is the fraction of cases for which the outcome is positive and correctly identified. Similarly, the specificity is the fraction of cases correctly identified as negative. A Receiver Operating Characteristic (ROC) (Fawcett, 2006) is the collection of all tuples of (specificity, sensitivity) obtained by varying a threshold $\vartheta$ on the quantity used as a predictor.

Formally, we consider a set of objects $S$ with a binary partition, i.e. subsets $S^{+}$and $S^{-}$with $S^{+} \cap S^{-}=\emptyset$ and $S^{+} \cup S^{-}=S$. As a predictor of this partition, we consider a function $v: S \rightarrow \mathbb{R}$ and a threshold value $\vartheta \in \mathbb{R}$. An object $x \in S$ is predicted as positive $(+)$ if $v(x) \geq \vartheta$. The sensitivity measures the fraction of objects from $S^{+}$predicted to be positive $(+)$, i.e.

$\frac{\left|\left\{x \in S^{+}: v(x) \geq \vartheta\right\}\right|}{\left|S^{+}\right|}$.

and analogously, the specificity is the fraction of objects from $S^{-}$predicted to be negative $(-)$,

$\frac{\left|\left\{x \in S^{-}: v(x)<\vartheta\right\}\right|}{\left|S^{-}\right|}$.

For the prediction of lethality, $S$ is the set of all single knock-outs in all network realizations considered, $S^{+}$are the lethal knock-outs, $S^{-}$the viable ones. Analogously in the context of double knock-outs, $S^{+}$are the synthetically lethal cases. The function $v$ is the predictor used, such as out-degree, in-degree etc. ROC plots show sensitivity as a function of specificity subtracted from 1 .
Acknowledgments This work has been funded by VolkswagenStiftung through the initiative on Complex Networks as Phenomena across Disciplines.

\section{References}

Albert R, Jeong H, Barabási AL, 2000. Error and attack tolerance of complex networks. Nature 406:378-382.

Albert R, Othmer HG, 2003. ... but no kinetic details needed. SIAM News 36.

Aldana M, Balleza E, Kauffman S, Resendiz O, 2007. Robustness and evolvability in genetic regulatory networks. Journal of Theoretical Biology 245:433 448.

Aldana M, Coppersmith S, Kadanoff L, 2003. Boolean Dynamics with Random Couplings. Perspectives and Problems in Nonlinear Science A Celebratory Volume in Honor of Lawrence Sirovich (pp. 23-89).

Alon U, 2007. Network motifs: theory and experimental approaches. Nature Reviews Genetics 8:450-461.

Boldhaus G, Bertschinger N, Rauh J, Olbrich E, Klemm K, 2010. Robustness of Boolean dynamics under knockouts. Physical Review E 82:021916.

Boldhaus G, Klemm K, 2010. Regulatory networks and connected components of the neutral space. The European Physical Journal B 77:233-237.

Bornholdt S, 2005. Less is more in modeling large genetic networks. Science 310:449 - 451.

Bower JM, Bolouri H, editors, 2001. Computational Modeling of Genetic and Biochemical Networks. MIT Press.

Brandes U, 2001. A Faster Algorithm for Betweenness Centrality. Journal of Mathematical Sociology 25:163-177.

Buchler NE, Gerland U, Hwa T, 2003. On schemes of combinatorial transcription logic. Proceedings of the National Academy of Sciences 100:5136-5141.

Chan DA, Giaccia AJ, 2011. Harnessing synthetic lethal interactions in anticancer drug discovery. Nature Reviews Drug Discovery 8:437-449.

Davidich M, Bornholdt S, 2008. Boolean network model predicts cell cycle sequence of fission yeast. PLoS ONE 3:e1672.

de Visser J, Hermisson J, Wagner G, Meyers L, Bagheri H, Blanchard J, Chao L, Cheverud J, Elena S, Fontana W, Gibson G, Hansen T, Krakauer D, Lewontin R, Ofria C, Rice S, von Dassow G, Wagner A, Whitlock M, 2003. Perspective: Evolution and detection of genetic robustness. Evolution 57:19591972.

Derrida B, 1987. Dynamical phase transition in nonsymmetric spin glasses. Journal of Physics A Mathematical and General 20:721-725. 
Drossel B, 2008. Random Boolean Networks. In: Schuster HG, editor, Reviews of Nonlinear Dynamics and Complexity: Volume 1, chap. 3, (pp. 69-110). Weinheim: Wiley.

Fawcett T, 2006. An introduction to ROC analysis. Pattern Recognition Letters 27:861-874.

Freeman LC, 1977. A Set of Measures of Centrality Based on Betweenness. Sociometry 40:35-41.

Hartman JL, Garvik B, Hartwell L, 2001. Principles for the buffering of genetic variation. Science 291:10011004 .

Inger A, Solomon A, Shenhav B, Olender T, Lancet D, 2009. Mutations and lethality in simulated prebiotic networks. Journal of Molecular Evolution 69:568578.

Ispolatov I, Krapivsky PL, Yuryev A, 2005. Duplication-divergence model of protein interaction network. Physical Review E 71:1-22.

Jeong H, Mason SP, Barabási AL, Oltvai ZN, 2001. Lethality and centrality in protein networks. Nature 411:41-42.

Kauffman SA, 1969. Metabolic stability and epigenesis in randomly constructed genetic nets. Journal of Theoretical Biology 22:437-467.

Li F, Long T, Lu Y, Ouyang Q, Tang C, 2004. The yeast cell-cycle network is robustly designed. Proc Natl Acad Sci USA 101:4781-4786.

Luo JX, Turner MS, 2011. Functionality and metagraph disintegration in boolean networks. Journal of Theoretical Biology 282:65-70.

Pastor-Satorras R, Smith E, Sol RV, 2003. Evolving protein interaction networks through gene duplication. Journal of Theoretical Biology 222:199 - 210.

Solé RV, Pastor-Satorras R, Smith E, Kepler TB, 2002. A model of large-scale proteome evolution. Advances in Complex Systems .

Szejka A, Mihaljev T, Drossel B, 2008. The phase diagram of random threshold networks. New Journal of Physics 10:063009.

Wagner A, 1994. Evolution of gene networks by gene duplications: a mathematical model and its implications on genome organization. Proceedings of the National Academy of Sciences 91:4387-4391.

Wuchty S, Stadler PF, 2003. Centers of complex networks. J Theor Biol 223:45-53. 